\title{
Enhancing knowledge on positive living together with sexual and reproductive health among HIV infected adolescents on anti-retroviral therapy: A quasi- experimental study
}

\author{
Myo-Myo Mon *, Kyaw-Min Htut *, Phyo Aung-Naing *, San Hone ${ }^{\text {**, }}$, Htun-Nyunt Oo ** \\ * Department of Medical Research, Ministry of Health and Sports, Myanmar \\ ** National AIDS Program, Ministry of Health and Sports, Myanmar \\ DOI: 10.29322/IJSRP.10.10.2020.p10603 \\ http://dx.doi.org/10.29322/IJSRP.10.10.2020.p10603
}

\begin{abstract}
Information on positive living as well as sexual and reproductive health (SRH) is essential for HIV infected adolescents on anti-retroviral therapy (ART). There was very little intervention study among them and effects of intervention have not studied yet. A quasi-experimental study encompassing baseline assessment, intervention and post-intervention assessment after one month was done by applying a mixedmethods design at Mandalay and Magway in 2019. Current study aims to assess the effectiveness of the intervention in terms of knowledge and attitude on SRH and positive living. Intervention package included aspects of positive living incorporated with SRH information which were conducted in group sessions including body mapping, case scenarios and discussions. Flyers describing the messages on positive living and SRH were distributed at the end. Face-to-face interview with HIV infected adolescents on ART, focus group discussions with their guardians and key informant interviews with service providers were conducted. Descriptive statistics were shown accordingly and bivariate analysis was done using paired t-test and McNemar test. A total of 76 adolescents aged 10-15 years participated in the intervention. Mean age was $12.9 \pm 1.7$ years and $56.6 \%$ were male. Over $88 \%$ were attending the school and $53.9 \%$ were double orphans. At post-intervention assessment, overall SRH knowledge score was significantly increased $(19.8 \pm 8.7$ and $31.6 \pm 7.2, p<0.001$, total 44 items). Specifically, improved knowledge scores regarding puberty $(3.6 \pm 1.9$ and $6.3 \pm 1.6, \mathrm{p}<0.001)$, adolescent pregnancy $(3.1 \pm 1.5$ and $4.4 \pm 0.9, \mathrm{p}<0.001)$, contraception $(3.3 \pm 2.2$ and $6.9 \pm 2.5, \mathrm{p}<0.001)$, sexually transmitted infections $(2.2 \pm 1.3$ and $3.3 \pm 1.2, \mathrm{p}<0.001)$ and HIV $(7.5 \pm 3.8$ and $10.6 \pm 3.1, \mathrm{p}<0.001)$ were seen. Improved knowledge score on positive living was also seen (9.0 \pm 6.9 and $14.9 \pm 5.9, \mathrm{p}<0.001$, total 22 items). Proportions of adolescents with negative attitude on SRH was reduced at postintervention assessment $(27.6 \%$ and $9.2 \%, \mathrm{p}<0.01)$. Health care providers indicated that provision of positive living and SRH information is one of the gaps in transitional care of HIV infected adolescents. Providers desired to extend the intervention by using the flyers at their ART clinics. Improved knowledge on positive living and SRH among HIV infected adolescents was achieved by applying the simple intervention package. Sustainability at the
\end{abstract}

Keywords - HIV infected adolescents, sexual and reproductive health, positive living, quasi-experimental, Myanmar

\section{INTRODUCTION}

Globally, adolescents living with HIV are a growing cohort and most of them acquired HIV infection vertically from their parents. On the other hand, some adolescents got infection from other routes during their childhood or early adolescence. With the increasing use of antiretroviral therapy (ART), children with vertically acquired HIV infection are reaching adolescence. In 2018, there were approximately 1.6 million adolescents aged 1019 years living with HIV worldwide. ${ }^{1}$ Adolescence represents the transition period from childhood to adulthood with a significant puberty changes leading to complete physical, mental and sexual maturity. ${ }^{2}$

Previous studies have documented the presence of unmet need for sexual and reproductive health (SRH) information, ${ }^{3,4}$ sexual debut among children under the care of pediatric health service, ${ }^{5}$ and unmet need for contraception. ${ }^{6}$ A review on comprehensive SRH education found that adolescents aged 10 to 14 years were at highrisk of adopting reproductive risk behaviors and suffered from the reproductive health consequences. ${ }^{7}$ The interventions addressing SRH in early adolescents (10-14 years) were recommended to be the priority issues. ${ }^{8}$

Living positively with HIV includes receiving health care, taking care of sexual health (e.g- safe sex practice), staying active and socially engaged, taking enough rest, taking care of personal hygiene and nutrition, and avoiding unhealthy behaviors (alcohol drinking, smoking, drug use, unsafe sex, etc). In general, positive living includes keeping one's mind healthy, one's body healthy, one's soul healthy and living responsibly with HIV and preventing new HIV infections. ${ }^{9}$

Previous intervention study in Myanmar had documented that mindfulness-integrated reproductive health package was effective for improving SRH knowledge and psychological behavior of HIV infected and affected adolescents. ${ }^{10,11}$ One of the specific objectives of the Strategic Plan on Reproductive Health (20142018 ) is to expand reproductive health information and services for adolescents and youths. ${ }^{12}$ Information related to 
adolescents before referring to adult ART site. Current intervention package includes providing SRH information incorporated with aspects of positive living. Intervention activities were conducted in group sessions with active participation of adolescents. Yet, there was no intervention study focusing on positive living and SRH together. Therefore, current study was conducted with the aim of assessing the effectiveness of intervention on sexual and reproductive health and positive living among HIV infected adolescents on ART. Specific objectives were 1) to identify and compare the awareness, knowledge and attitude regarding SRH and positive living among HIV infected adolescents on ART before and after the intervention, and 2) to determine the perspectives of health care providers and guardians regarding the intervention. The Findings from current intervention study would become input in planning comprehensive care of HIV infected adolescents before transferring to adult site.

\section{METHODOLOGY}

Study design, area and population

A quasi-experimental study encompassing baseline assessment, intervention and post-intervention assessment after one month by applying a mixed-methods design was conducted in Mandalay and Magway. HIV infected adolescents on ART, their guardians and service providers were the study populations.

Inclusion criteria: HIV infected adolescents on ART aged 10-15

Exclusion criteria: 1) HIV infected adolescents on ART who have intellectual disabilities according to the information from providers and guardians, 2) HIV infected adolescents on ART who are hospitalized or bed-ridden

\section{Sample size and sampling}

Considering the proportion of adolescents having good SRH knowledge among HIV infected adolescents as $40 \%$ and at least $35 \%$ of knowledge improvement after the intervention, precision 0.05 , power $80 \%$, and loss to follow-up $5 \%$, required sample size was 76. Purposive sampling was applied and adolescents were recruited through Myanmar Positive Group (MPG) and peer counselors from public ART centers. For qualitative data collection, two focus group discussions (FGDs) with selected guardians and four key informant interviews (KIIs) with service providers were done.

\section{Intervention package}

Intervention package was developed by adapting the SRH messages for adolescents and module of training on positive living.9 The package included information on positive living and information on sexual and reproductive health specific to the adolescents. Adolescents had to attend the group session which lasted approximately 3-4 hours. The group sessions were conducted separately for each gender and moderated by principal investigator and co-investigator.

Components of "Positive Living"

- Prevent further transmission of HIV

- Receive regular health care
- Stay active \& socially engaged

- Take enough rest \& nutrition

- Take care of personal hygiene

- Take care of sexual health

- Avoid unhealthy behaviour: Alcohol, Smoking, Drug use

Components of "Sexual and Reproductive Health"

- Puberty: Physiological changes, Menstrual cycle

- Adolescent pregnancy and its consequences

- Contraception

- HIV

- STI

\section{Data collection procedure and tools}

Training of interviewers was done at Department of Medical Research before intervention activities and assessments. Baseline assessment was conducted to explore the adolescents' knowledge and attitude on SRH and positive living before the intervention. Intervention activities were conducted applying group discussion, case scenarios and health education sessions. Evaluation of the outcomes was done at post assessment after one month by both quantitative and qualitative methods. Face-to-face interviews were done with adolescents using a structured questionnaire. Focus group discussions (FGD) and key informant interviews (KII) were also conducted with guardians and service providers.

\section{Data management and analysis}

Data entry and analysis of quantitative data was done using EpiData 3.1 and SPSS version 21. Descriptive information was described by using frequency or mean/median as appropriate. Responses on knowledge questions were given a score of " 1 " for correct answer and " 0 " for incorrect answer. Total knowledge score was calculated for each SRH component and also for total SRH questions. Possible total knowledge score for SRH ranged from "0" to "44". Similarly, total knowledge score was calculated for question on positive living and possible total score ranged from " 0 " to " 22 ". Response on each attitude question was given a score of " 1 " to " 5 " depending on the direction of the statement whereas having positive attitude got higher score. Total attitude score was calculated and possible scores ranged from "9" to " 45 ". Then, it was categorized into "negative attitude" and "positive attitude" by using the cut-off value of " 27 " since neutral value for each item was " 3 " and there were "9" attitude items. Comparisons of outcomes between baseline and end-line assessments were done by using paired " $\mathrm{t}$ " test, McNemar test and student " $\mathrm{t}$ " test. Qualitative information was analyzed by using manual thematic analysis.

\section{Ethical consideration}

Informed consent from the guardians and assent from the adolescents were requested after thorough explanation about the study's objectives. Confidentiality and anonymity of the participants' information was strictly maintained. Ethical approval was obtained from Ethics Review Committee of Department of Medical Research. Informed consents were also taken from all the guardians and providers before FGD and KII.

Expected applicability 
Evidence based information resulted from current intervention could be applied for improving comprehensive care of HIV infected adolescents. Responsible service providers

\section{FINDINGS}

A total of 76 adolescents aged 10-15 years participated in the intervention. Table (1) shows the background characteristics of adolescents on ART. Their mean age was $12.9 \square 1.7$ years and $56.6 \%$ were male. Over $88 \%$ were attending the school and $53.9 \%$ were double orphans.

Table (1) Background characteristics of HIV infected adolescents on ART

\begin{tabular}{|c|c|c|}
\hline Characteristics & Number & Percentage \\
\hline \multicolumn{3}{|l|}{ Gender } \\
\hline Male & 43 & 56.6 \\
\hline Female & 33 & 43.4 \\
\hline \multicolumn{3}{|l|}{ Age } \\
\hline Mean \pm SD & \multicolumn{2}{|c|}{$12.9 \pm 1.7$} \\
\hline Min-Max & \multicolumn{2}{|c|}{$10-15$} \\
\hline \multicolumn{3}{|l|}{ Schooling } \\
\hline Out of school & 9 & 11.8 \\
\hline $\begin{array}{l}\text { Currently attending } \\
\text { school }\end{array}$ & 67 & 88.2 \\
\hline \multicolumn{3}{|l|}{ Parental status } \\
\hline Both parents alive & 14 & 18.4 \\
\hline Paternal orphan & 11 & 14.5 \\
\hline Maternal orphan & 10 & 13.2 \\
\hline Double orphan & 41 & 53.9 \\
\hline
\end{tabular}

As shown in Figure (1), considerably higher proportions of adolescents could mention the male and female puberty changes at post-intervention than at baseline. Highest proportional difference between two assessments was seen regarding the knowledge on sperm production at puberty $(17.1 \%$ and $71.1 \%)$.

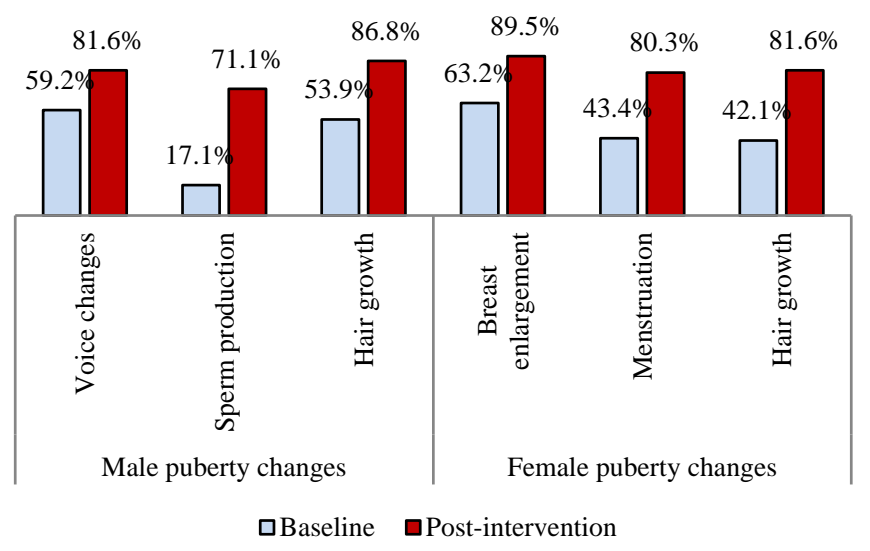

Figure (1) Knowledge of HIV infected adolescents on male and female puberty changes at baseline and post-intervention assessments $(n=76)$

Comparison of knowledge scores and attitude on SRH and positive living among HIV infected adolescents on ART at acknowledged the intervention and expressed the desire to continue at their ART clinics.

baseline and post-intervention is described in Table (2). At postintervention assessment, substantial increase in total knowledge score on SRH was seen in comparing to baseline (19.8 \pm 8.7 and $31.6 \pm 7.2, \mathrm{p}=0.0001$, total items: 44). As shown in the table, improved scores were detected for all SRH topics at postintervention assessment. Likewise, considerable improvement in knowledge on positive living was detected $(9.0 \pm 6.9$ and $14.9 \pm 5.9$, $\mathrm{p}=0.0001$, total items: 22). Additionally, proportion of adolescents with negative attitude on SRH significantly reduced at post-intervention $(27.6 \%$ and $9.2 \%, \mathrm{p}=0.007)$.

Table (2) Comparison of knowledge scores and attitude on SRH and positive living among HIV infected adolescents on ART at baseline and post-intervention

\begin{tabular}{|c|c|c|c|}
\hline Characteristics & $\begin{array}{c}\text { Baseline } \\
\mathrm{N}=76\end{array}$ & $\begin{array}{c}\text { Post- } \\
\text { intervention } \\
N=76\end{array}$ & p-value \\
\hline Total SRH knowledge & & & $0.0001^{\mathrm{a}}$ \\
\hline Mean \pm SD & $19.8 \pm 8.7$ & $31.6 \pm 7.2$ & \\
\hline Min-Max & $1-36$ & $13-44$ & \\
\hline \multicolumn{4}{|l|}{ Knowledge on SRH topic } \\
\hline Puberty & $3.6 \pm 1.9$ & $6.3 \pm 1.6$ & $0.0001^{\mathrm{a}}$ \\
\hline Adolescent pregnancy & $3.1 \pm 1.5$ & $4.4 \pm 0.9$ & $0.0001^{\mathrm{a}}$ \\
\hline Contraception & $3.2 \pm 2.2$ & $6.9 \pm 2.5$ & $0.0001^{\mathrm{a}}$ \\
\hline STI & $2.2 \pm 1.3$ & $3.3 \pm 1.2$ & $0.0001^{\mathrm{a}}$ \\
\hline HIV & $7.5 \pm 3.7$ & $10.6 \pm 3.0$ & $0.0001^{\mathrm{a}}$ \\
\hline Attitude on SRH (n,\%) & & & $0.007^{\mathrm{b}}$ \\
\hline Negative attitude & $21(27.6)$ & $7(9.2)$ & \\
\hline Positive attitude & $55(72.4)$ & $69(90.8)$ & \\
\hline $\begin{array}{l}\text { Knowledge on positive } \\
\text { living }\end{array}$ & & & $0.0001^{\mathrm{a}}$ \\
\hline Mean \pm SD & $9.0 \pm 6.9$ & $14.9 \pm 5.9$ & \\
\hline Min-Max & $0-22$ & $3-22$ & \\
\hline $\begin{array}{l}\text { Attitude on positive } \\
\text { living }(\mathrm{n}, \%)\end{array}$ & & & $1.0^{\mathrm{b}}$ \\
\hline Negative attitude & $11(14.5)$ & $11(14.5)$ & \\
\hline Positive attitude & $65(85.5)$ & $65(85.5)$ & \\
\hline
\end{tabular}

a paired t-test; ${ }^{\mathrm{b}} \mathrm{McNemar}$ test

STI: Sexually Transmitted Infections

As shown in Table (3), gender difference in change in knowledge score was seen and the change was higher in male than female adolescents $(13.9 \pm 8.4$ and $8.9 \pm 8.1, \mathrm{p}=0.01)$. Over $98 \%$ satisfied on the intervention and $80 \%$ of them interested more on the positive living than SRH. 
Table (3) Comparison of change in knowledge scores on SRH and positive living among male and female HIV infected adolescents on ART

\begin{tabular}{lccc}
\hline Characteristics & $\begin{array}{c}\text { Male } \\
\text { adolescents } \\
\mathbf{N}=\mathbf{4 3}\end{array}$ & $\begin{array}{c}\text { Female } \\
\text { adolescents } \\
\mathbf{N}=\mathbf{3 3}\end{array}$ & $\begin{array}{c}\mathbf{p -} \\
\text { value }^{\mathbf{c}}\end{array}$ \\
\hline $\begin{array}{l}\text { Knowledge score } \\
\text { change on SRH } \\
\text { (Mean } \pm \text { SD) }\end{array}$ & $13.9 \pm 8.4$ & $8.9 \pm 8.1$ & 0.01 \\
\hline $\begin{array}{l}\text { Knowledge score } \\
\text { change on positive }\end{array}$ & & & 0.13 \\
$\begin{array}{l}\text { living } \\
(\text { Mean } \pm \text { SD) }\end{array}$ & $6.8 \pm 6.4$ & $4.7 \pm 5.0$ & \\
\hline
\end{tabular}

$\mathrm{c}$ t-test

During key informant interviews, the service providers pointed out the importance of filling the gap in comprehensive care of HIV infected adolescents. They highlighted that current intervention could provide essential information for the infected adolescents. According to the providers, though SRH information was critical for the HIV infected adolescents, there was no specific SRH service provided for them. Providers acknowledged that discussion on concept of positive living especially about the health risk behaviors was beneficial for the infected adolescents.

Some of their expressions were as follows:

"Discussion is good as it includes about risk behaviors like smoking, alcohol drinking under the topic of positive living. At that age, it would become primordial prevention. They have already experienced from systemic effects of ART. Thanks for telling about those things since it is the best if they could avoid smoking and alcohol drinking" (Responsible provider from Mandalay)

"Though I know that SRH information should be provided I'm not sure what kind of information we should discuss at what age..." (Responsible provider from Magway)

"We could produce vinyl poster from the information of this flyer... and train our peers. And then peers can disseminate the information to the adolescents at the time of ART visit. We can also distribute the flyers... we could continue like that after the intervention" (Responsible provider from Magway)

During focus group discussion with parents/guardians, many of them agreed with the intervention package on positive living and SRH. Most appropriate age for provision of SRH knowledge was discussed and many of them indicated "13-14 years" while some of them mentioned as " 10 years".

"I think, 13-14 years is appropriate to learn SRH information since they could understand at that age" (46 years old mother)

"I think, one should know about reproductive health around 10 years of age... since they are using mobile phones and they may also know from others..." (34 years old mother)

This publication is licensed under Creative Commons Attribution CC BY

http://dx.doi.org/10.29322/IJSRP.10.10.2020.p10603
"It's better to talk by the providers than the parents since they could learn more knowledge. It's also good that training is held on school holidays..." (34 years old mother and 67 years old grandmother)

\section{DISCUSSIONS}

Current intervention package tried to fill an important gap in comprehensive care of HIV infected adolescents on ART by enhancing their knowledge on positive living and SRH. Providers acknowledged the benefits of intervention and showed their willingness to continue the intervention.

Positive living is a lifestyle which should be adopted by an HIVinfected person to live life as fully as possible and to improve the quality of life of people living with HIV. ${ }^{9}$ At post-intervention assessment, adolescents' knowledge on positive living improved significantly which showed the effectiveness of intervention. Improved knowledge would lead to positive practices in their adult life. If the HIV infected adolescents lived positively and responsibly, then it could help in reducing further transmission of HIV.

Likewise, improved knowledge and attitude on SRH was seen at post-intervention assessment. Evidences had shown that HIV infected adolescents have SRH needs and they are at risk of engaging in sexual risk behaviors. ${ }^{13,14} \mathrm{~A}$ review of the intervention studies on SRH among young people living with HIV identified that most interventions used cognitive behavioral therapy and/or psychosocial support resulted in positive SRH behavioral outcomes. The review also highlighted that there have been a limited number of evaluated interventions to strengthen SRH of young people living with HIV. ${ }^{15}$ Though there were some intervention studies, comparison of effectiveness of these intervention studies was difficult since different studies applied various intervention approaches focusing on different issues of SRH.

In Thailand, a clinic-based intervention program on HIV infected adolescents through group and individual sessions was well accepted by the adolescents and was able to improve their knowledge and attitude. The program focused on sexual risk reduction, health knowledge, coping skills and life goals. ${ }^{16} \mathrm{~A}$ study on sexual health programming among HIV positive adolescents in South Africa had documented that provision of social protection could reduce unprotected sex. ${ }^{17}$

During qualitative discussions with service providers, they pointed out the lack of specific SRH intervention and services as the important gap in comprehensive care of HIV infected adolescents. Similarly, previous studies in India and African countries had documented the health care needs and service delivery gaps for HIV infected adolescents such as integrated comprehensive SRH services, standardized transition and quality counseling. ${ }^{18,19}$

Certain strengths of current intervention should be documented. This was the first evaluated intervention study with integrated package of positive living and SRH information for adolescents living with HIV. It applied simple participatory methods like body mapping, two-way discussions and case scenarios. Flyers of summary information were also distributed as a training material to enhance their understanding. Besides strengths, there were certain limitations that should be acknowledged. Lack of control group might have some drawbacks that we could not compare the effects of intervention with the non- 
intervention group. Furthermore, we did not identify other sources of SRH information among the adolescents.

\section{CONCLUSION}

Improved knowledge on positive living and SRH among HIV infected adolescents was achieved by applying the simple intervention package which could be easily utilized in ART clinic settings. Sustainability at the study sites and expansion to other areas should be considered.

\section{ACKNOWLEDGMENT}

The study was conducted with the funding support of Implementation Research Grant of Ministry of Health and Sports and grant from Department of Medical Research, Myanmar. We would like to express our thanks to Prof. Dr. Zaw Than Htun, Director General, Department of Medical Research for giving permission to conduct the study. We also wish to extend our thanks to the responsible persons from National AIDS Program, focal persons from Myanmar Positive Group and all the adolescents included in the study.

\section{REFERENCES}

[1] UNICEF. UNICEF Data: Monitoring the situation of children and women [Internet]. 2019. Available from: https://data.unicef.org/topic/hivaids/adolescents-young-people/, accessed 21 November 2019.

[2] WHO | Adolescent development [Internet]. WHO. [cited 2014 Dec 1]. Available from: http://www.who.int/maternal_child_adolescent/topics/adolescence/develop ment/en/

[3] Birungi H, Obare F, Mugisha JF, Evelia H, Nyombi J. Preventive service needs of young people perinatally infected with HIV in Uganda. AIDS Care. 2009 Jun;21(6):725-31.

[4] Hodgson I, Ross J, Haamujompa C, Gitau-Mburu D. Living as an adolescent with HIV in Zambia--lived experiences, sexual health and reproductive needs. AIDS Care. 2012;24(10):1204-10.

[5] Croucher AP, Jose S, McDonald S, Foster C, Fidler S. Sexual and reproductive health in a UK cohort of young adults perinatally infected with HIV. Sexually Transmitted Infections. 2013 Aug;89(5):392-4.

[6] Robinson JA, Fox MC, Jamshidi R, Trent M, Anderson J, Burke AE. Contraceptive needs of human immunodeficiency virus-positive adolescent women compared with a human immunodeficiency virus-negative cohort. Obstet Gynecol. 2014 May;123 Suppl 1:13S.

[7] Haberland N, Rogow D. Sexuality Education: Emerging Trends in Evidence and Practice. J Adolesc Health. 2015 Jan;56(1):S15-21.

[8] Chandra-Mouli V, Svanemyr J, Amin A, Fogstad H, Say L, Girard F, et al. Twenty Years After International Conference on Population and Development: Where Are We With Adolescent Sexual and Reproductive Health and Rights? J Adolesc Health 2015 Jan;56(1):S1-6.

[9] ICAP, Columbia University, Milman School of Public Health. Adolescent HIV care and treatment: A training curriculum for health workers, Participant Manual. 2012

[10] Myo-Myo Mon, Tippawan Liabsuetrakul, Edward B. McNeil and Kyaw-Min Htut. Mindfulness-integrated reproductive health package for adolescents with parental HIV infection: A group randomized-controlled trial. Vulnerable Children and Youth Studies 2017; 12:2, 147-159.

[11] Myo-Myo Mon, Tippawan Liabsuetrakul and Kyaw-Min Htut. Effectiveness of mindfulness intervention on psychological behaviors among adolescents with parental HIV infection: A group-randomized controlled trial. Asia Pacific Journal of Public Health. 2016 Nov;28(8):765-775.

[12] Department of Health, Ministry of Health, Myanmar. Five year strategic plan for reproductive health (2014-2018)

[13] Ndongmo TN, Ndongmo CB \& Michelo C. Sexual and reproductive health knowledge and behavior among adolescents living with HIV in Zambia: a case study. Pan African Medical Journal. 2017 Feb 20; $26: 71$.

[14] Okawa S, Mwanza-Kabaghe S, Mwiya M, Kikuchi K, Jimba M, Kankasa C, et al. Sexual and reproductive health behavior and unmet needs among a sample of adolescents living with HIV in Zambia: a cross-sectional study. Reproductive Health. 2018 Mar27;15(1):55.

[15] Pretorius L, Gibbs A, Crankshaw T \& Willan S. Interventions targeting sexual and reproductive health and rights outcomes of young people living with HIV: a comprehensive review of current interventions from sub-Saharan Africa. Global Health Action. 2015 Nov 2;8:28454.

[16] Chokephaibulkit K, Tarugsa J, Lolekha R, Leowsrisook P, Manaboriboon B, Naiwatanakul T, et al. Outcomes of a comprehensive youth program for HIVinfected adolescents in Thailand. Journal of the Association of Nurses in AIDS Care. 2015 Nov;26(6):758-769.

[17] Toska E, Cluver LD, Boyes ME, Isaacsohn M, Hodes R \& Sherr L. School, supervision and adolescent-sensitive clinic care: Combination social protection and reduced unprotected sex among HIV-positive adolescents in South Africa. AIDS Behaviour. 2017 Sep;21(9):2746-2759.

[18] Mark D, Armstrong A, Andrade C, Penazzato M, Hatane L, Taing L, et al HIV treatment and care services for adolescents: a situational analysis of 218 facilities in 23 sub-Saharan African countries. Journal of the International AIDS Society. 2017 May16;20(Suppl 3):21591.

[19] Verma A, Sahay S. Healthcare needs and programmatic gaps in transition from pediatric to adult care of vertically transmitted HIV infected adolescents in India. PLoS One. 2019 Oct 29;14(10):e0224490.

\section{AUTHORS}

First Author - Myo-Myo Mon, MBBS, MSc (Public Health), MSc (Epidemiology), PhD (Epidemiology), Department of Medical Research, Ministry of Health and Sports, Myanmar, mmyomon@gmail.com

Second Author - Kyaw-Min Htut, MBBS, Department of Medical Research, Ministry of Health and Sports, Myanmar, 84kyawminhtut@gmail.com

Third Author - Phyo Aung-Naing, MBBS, MPH, Department of Medical Research, Ministry of Health and Sports, Myanmar, phyoaungnaing84@gmail.com

Fourth Author - San Hone, MBBS, MPH, PhD, National AIDS Program, Ministry of Health and Sports, Myanmar,

sanhone@ucla.edu

Fifth Author - Htun-Nyunt Oo, MBBS, MPH, National AIDS

Program, Ministry of Health and Sports, Myanmar, dr.tunnyuntoo@gmail.com

Correspondence Author - Myo-Myo Mon, Department of Medical Research, Ministry of Health and Sports, Myanmar, mmyomon@gmail.com 